\title{
Evaluation of Bi-Layer Silk Fibroin Grafts for Tubular Ureteroplasty in a Porcine Defect Model
}

\author{
Gokhan Gundogdu ${ }^{1}$, Zhamshid Okhunov ${ }^{1}$, Vivian Cristofaro ${ }^{2,3}$, Stephanie Starek ${ }^{1}$, \\ Faith Veneri ${ }^{1}$, Hazem Orabi ${ }^{1}$, Pengbo Jiang ${ }^{1}$, Maryrose P. Sullivan ${ }^{2,3}$ and \\ Joshua R. Mauney ${ }^{1,4 *}$
}

${ }^{1}$ Department of Urology, University of California, IIvine, IIvine, CA, United States, ${ }^{2}$ Division of Urology, Veterans Affairs Boston Healthcare System, Boston, MA, United States, ${ }^{3}$ Department of Surgery, Brigham and Women's Hospital, Harvard Medical School, Boston, MA, United States, ${ }^{4}$ Department of Biomedical Engineering, University of California, Irvine, Irvine, CA, United States

\section{OPEN ACCESS}

Edited by:

Joaquim Miguel Oliveira, University of Minho, Portugal

Reviewed by: Viviana Pinto Ribeiro University of Minho, Portugal Mariana R. Carvalho, I3Bs - University of Minho, Portugal

*Correspondence: Joshua R. Mauney mauneyj@hs.uci.edu

Specialty section: This article was submitted to Tissue Engineering and Regenerative Medicine,

a section of the journal Frontiers in Bioengineering and Biotechnology

Received: 10 June 2021 Accepted: 31 August 2021 Published: 17 September 2021

Citation:

Gundogdu G, Okhunov Z, Cristofaro V, Starek S, Veneri $F$, Orabi $H$, Jiang $P$, Sullivan MP and Mauney JR (2021) Evaluation of Bi-Layer Silk Fibroin Grafts for Tubular Ureteroplasty in a Porcine Defect Model. Front. Bioeng. Biotechnol. 9:723559. doi: 10.3389/fbioe.2021.723559
Ureteral reconstruction with autologous tissue grafts is often limited by tissue availability and donor site morbidity. This study investigates the performance of acellular, bi-layer silk fibroin (BLSF) scaffolds in a porcine model of ureteroplasty. Tubular ureteroplasty with BLSF grafts in combination with transient stenting for 8 weeks was performed in adult female, Yucatan, mini-swine ( $N=5)$. Animals were maintained for 12 weeks post-op with imaging of neoconduits using ultrasonography and retrograde ureteropyelography carried out at 2 and 4 weeks intervals. End-point analyses of ureteral neotissues and unoperated controls included histological, immunohistochemical $(\mathrm{IHC})$, histomorphometric evaluations as well as ex vivo functional assessments of contraction/relaxation. All animals survived until scheduled euthanasia and displayed mild hydronephrosis (Grades 1-2) in reconstructed collecting systems during the 8 weeks stenting period with one animal presenting with a persistent subcutaneous fistula at 2 weeks post-op. By 12 weeks of scaffold implantation, unstented neoconduits led to severe hydronephrosis (Grade 4) and stricture formation in the interior of graft sites in $80 \%$ of swine. Bulk scaffold extrusion into the distal ureter was also apparent in $60 \%$ of swine contributing to ureteral obstruction. However, histological and $\mathrm{IHC}$ analyses revealed the formation of innervated, vascularized neotissues with a-smooth muscle actin+ and SM22a+ smooth muscle bundles as well as uroplakin $3 \mathrm{~A}+$ and pan-cytokeratin + urothelium. Ex vivo contractility and relaxation responses of neotissues were similar to unoperated control segments. BLSF biomaterials represent emerging platforms for tubular ureteroplasty, however further optimization is needed to improve in vivo degradation kinetics and mitigate stricture formation.

Keywords: biomaterials, silk fibroin, tissue engineering, ureter, scaffold

\section{INTRODUCTION}

Ureteral injuries caused by intraoperative endourological maneuvers, radiation exposure, or gynecological procedures can lead to life-threatening complications such as sepsis, urinary tract obstruction, and loss of renal function (Packiam et al., 2016; Blackwell et al., 2018). In addition, penetrating traumas from gunshot and stab wounds can also result in ureteral perforation with a 
mortality rate of $6 \%$ (Siram et al., 2010). Surgical management of short ureteral defects $(<2 \mathrm{~cm}$ in length) is often performed with primary anastomosis following resection of damaged segments (Burks and Santucci, 2014), however for long ureteral pathologies, autologous tissue grafting is necessary to restore organ continuity (Mauck et al., 2011; Kocot et al., 2017). Traditional surgical approaches for ureteral repair deploying patient-derived donor tissues include uretero-ureterostomy, uretero-neocystostomy, Boari flap, transuretero-ureterostomy, renal autotransplantation, and ileal interposition with the choice of method being dependent on the size and location of injury (Simaioforidis et al., 2013). However, the success of these strategies can be compromised by donor site morbidity, reflux, stricture formation, hydronephrosis, and persistent anastomotic leakage (Engel et al., 2015; Xiong et al., 2020; Heijkoop and Kahokehr, 2021). These complications often necessitate secondary salvage procedures to maintain upper urinary tract function, but in the worst case scenario, nephrectomy may be required (Onal et al., 2018; Vasudevan et al., 2019). These reports highlight the need for novel strategies for ureteral reconstruction which can minimize unwanted side-effects and promote functional tissue regeneration.

Biodegradable 3-D scaffolds composed of decellularized tissues, collagen or synthetic polyesters have been previously investigated as potential substitutes to autologous tissue grafts for ureteral repair (Kloskowski et al., 2013; Simaioforidis et al., 2013; de Jonge et al., 2015). These biomaterials have been utilized alone or seeded with primary urothelial, smooth muscle, and/or mesenchymal stem cell populations for reconstruction of patch or tubular ureteral defects in preclinical animal models (El-Hakim et al., 2005; Liao et al., 2013; Meng et al., 2015; Xu et al., 2020). Despite the ability of these tissue engineered constructs to support constructive remodeling following onlay ureteroplasty (Liatsikos et al., 2001; Smith et al., 2002), suboptimal outcomes including stricture formation, hydronephrosis, graft contracture, and fibrosis have been reported following implantation of tubular scaffold configurations (Duchene et al., 2004; Janke et al., 2019). Currently, there is no FDA-approved tissue engineered device for ureteral tissue replacement. Advancements in ureteral tissue engineering are dependent on new scaffold designs which can preserve upper urinary tract function while promoting host regenerative responses.

Acellular, bi-layer silk fibroin (BLSF) grafts represent emerging options for urinary tract reconstruction due to their low immunogenicity, robust mechanical strength and elasticity, tunable biodegradability, and diverse processing plasticity (Sack et al., 2016). These protein-based, biomaterials derived from Bombyx mori silkworm cocoons can be fashioned into sheets or tubes and their bi-layer architecture prevents urinary extravasation at implant sites via a fluid-tight film layer, while a porous foam compartment allows for ingrowth of surrounding host tissues (Algarrahi et al., 2018a; Affas et al., 2019). In comparison to silk fibroin foams alone, the addition of the annealed film layer minimizes graft perforation and enhances suture retention strength during urinary tract reconstruction by increasing the ultimate tensile strength and elastic modulus of the bulk matrix (Tu et al., 2013). Previous reports from our laboratory have detailed the ability of BLSF grafts to promote improved functional performance, enhanced tissue regeneration, and minimal inflammatory reactions in respect to conventional SIS matrices across various preclinical models of bladder and urethral reconstruction encompassing both traumatic injury and pathologic settings (Chung et al., 2014a; Chung et al., 2014b). In addition, constructive remodeling of BLSF scaffolds has been shown to promote formation of innervated, vascularized urologic neotissues with contractile properties similar to native counterparts (Seth et al., 2013; Tu et al., 2013; Affas et al., 2019). In the present study, we evaluated the efficacy of tubular BLSF grafts to support functional tissue regeneration of ureteral defects in a porcine model of ureteroplasty.

\section{MATERIALS AND METHODS}

\section{Biomaterials}

Aqueous silk fibroin solutions derived from B. mori silkworm cocoons were used to fabricate BLSF biomaterial sheets following previously described protocols (Seth et al., 2013). Briefly, an 8\% weight/volume silk fibroin solution was dried in a casting vessel under a laminar flow hood for $48 \mathrm{~h}$ to produce a silk fibroin film. A $6 \%$ weight/volume silk fibroin solution was then combined with sieved granular $\mathrm{NaCl}(500-600 \mu \mathrm{M}$, average crystal diameter) in a ratio of $2 \mathrm{~g} \mathrm{NaCl}$ per $\mathrm{ml}$ of silk fibroin solution and layered on top of the silk fibroin film. The resultant solution was allowed to solidify and fuse to the silk fibroin film for $48 \mathrm{~h}$ at $37^{\circ} \mathrm{C}$ to create the BLSF matrix. $\mathrm{NaCl}$ was removed thereafter by washing the scaffold for $72 \mathrm{~h}$ in distilled water with regular volume changes. Structural and mechanical properties of BLSF matrices have been published in past reports (Algarrahi et al., 2018b). In particular, the BLSF biomaterial was composed of a foam compartment consisting of interconnected pores $(\sim 400 \mu \mathrm{m}$ diameter) buttressed by a homogeneous, non-porous film layer ( $\sim 200 \mu \mathrm{m}$ thick). Uniaxial tensile properties of the BLSF graft were previously determined as ultimate tensile strength: $0.3 \pm 0.1$ $\mathrm{MPa}$; elastic modulus: $3.6 \pm 1.3 \mathrm{MPa}$; and elongation to failure: $24.7 \pm 8.9 \%$ (Algarrahi et al., 2018b). BLSF matrix sheets were fashioned into tubular implants $(2 \mathrm{~cm}$ in length, $1 \mathrm{~cm}$ in outer diameter, $0.6 \mathrm{~cm}$ in inner diameter) using 4-0 propylene sutures. Biomaterials were steam sterilized in an autoclave at $120^{\circ} \mathrm{F}$ for 20 min prior to implantation.

\section{Surgical Procedures}

All animal husbandry, surgical manipulations, and imaging evaluations followed guidelines prescribed by the National Institutes of Health's Guidelines for the Care and Use of Laboratory Animals and were reviewed and approved by the University of California, Irvine Animal Care and Use Committee and performed under protocol AUP-19-166. This study was also conducted in compliance with ARRIVE guidelines (https:// arriveguidelines.org).

Tubular ureteroplasty was performed in five adult female, Yucatan mini-swine ( 24 weeks of age, $30-40 \mathrm{~kg}$, Premier BioSource, Ramona, CA) using either a retroperitoneal flank $(N=4)$ or transperitoneal $(N=1)$ approach (Figure 1). Male 

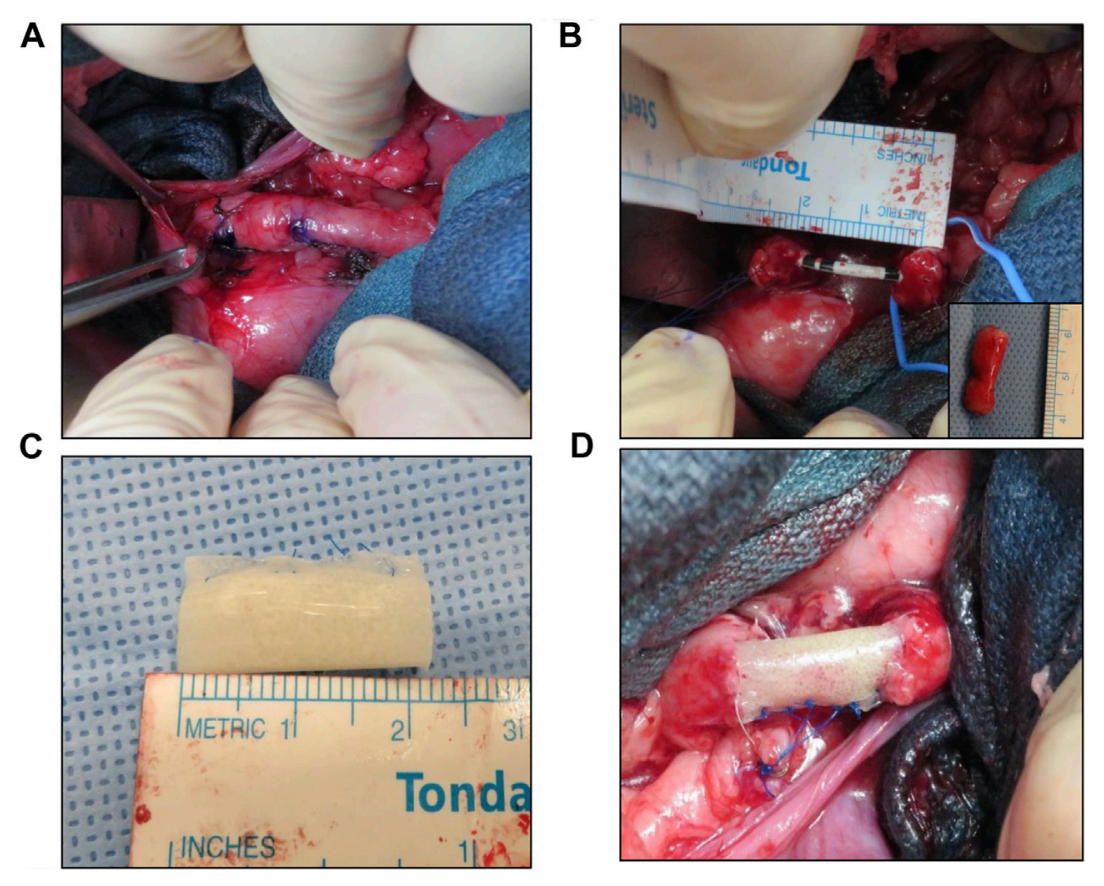

FIGURE 1 | Porcine tubular ureteroplasty model. (A) Exposure of proximal ureter and marking of target reconstructive area. (B) Creation of tubular ureteral defect and stent placement. Inset denotes excised tissue. (C) Tubular BLSF graft prior to implantation. (D) BLSF graft anastomosed into ureteral defect. BLSF, bi-layer silk fibroin scaffold.

swine were excluded from the study since catheter placement into the urinary tract cannot be performed reliably due to excessive tortuosity of the male urethra. Prior to operative procedures, swine were fasted $12 \mathrm{~h}$ with free access to water. General anesthesia was initiated by intramuscular injection of $0.4 \mathrm{mg} / \mathrm{kg}$ atropine, $2.2 \mathrm{mg} / \mathrm{kg}$ Anased (Lloyd Inc.; IA, United States), and $4.4 \mathrm{mg} / \mathrm{kg}$ Telazol (Zoetis Inc.; Parsippany, NJ, United States), and maintained with $1-4 \%$ isoflurane inhalation through an endotracheal tube. A retrograde ureteropyelogram (RUPG) was first performed via catheterization to confirm the native anatomy of the distal right ureter. The catheter was left inside the ureter to aid in surgical manipulations.

Swine were maintained in the supine position for the transperitoneal approach or were repositioned to the left lateral decubitus for the retroperitoneal flank procedure. Following skin incision, the abdominal wall layers were separated allowing entry into the retroperitoneal space. A $2 \mathrm{~cm}$ segment of the right upper ureter $\sim 5 \mathrm{~cm}$ below the pelvi-ureteric junction was resected without compromising the ureteral vasculature. The ureteral catheter was then replaced by a six French, double pigtail ureteral stent (Inlay Optima; BARD Inc., Covington, GA, United States) using a hybrid guidewire (Boston Scientific, Boston, MA, United States). A tubularized BLSF implant $(2 \mathrm{~cm}$ in length) was positioned in line within the ureteral defect and over the ureteral stent. The graft was then anastomosed to the surrounding host tissues using interrupted 50 monofilament polyglactin sutures. Proximal and distal boundaries of the implant with host ureter were marked with non-absorbable 4-0 polypropylene sutures (Ethicon, Somerville, NJ, United States). In addition, proximal/distal radiopaque marking rings were placed adjacent to the implant site to identify the graft area during fluoroscopic evaluations. A Penrose drain was placed into the retroperitoneum in Pigs 2-5 to drain any excess fluid over the course of 7 days and removed thereafter. The abdominal incision was closed in separate layers and a Foley catheter was introduced into the bladder. The Foley catheter was fixed to the skin with non-absorbable sutures and removed after 5 days. For postoperative pain management, all animals received subcutaneous infiltration of $1-3 \mathrm{mg} / \mathrm{kg}$ Bupivacaine $\mathrm{HCl} \quad 0.25 \%$ (Hospira Inc.; Lake Forest, IL, United States) after abdominal wound closure, an intramuscular injection of $2 \mathrm{mg} / \mathrm{kg}$ Banamine (Merck Animal Health; Kenilworth, NJ, United States) and subcutaneous injection of $0.15 \mathrm{mg} / \mathrm{kg}$ Buprenorphine SR (ZooPharm; Laramie, WY, United States) for $72 \mathrm{~h}$. Animals were recovered in warm blankets until they became sternal. Enrofloxacin (Baytril, Bayer Healthcare LLC, KA, United States) was intramuscularly administered $(7.5 \mathrm{mg} / \mathrm{kg})$ prior to each invasive procedure and was continued orally for 4 days post-op.

Following 8 weeks post-op, the ureteral stent was removed from the right ureter (except for Pig 2) by using a flexible cystoscope (Model \#: 11272C; Karl Storz, Tuttlingen, Germany) and endoscopic grasper (Model\#:11,275 FE, Karl Storz, Tuttlingen, Germany) to evaluate neoureter and kidney function without urinary diversion. Longitudinal imaging evaluations were performed prior to surgical manipulations and at $1,2,4,8$, and 12 weeks post-op to monitor ureter 
TABLE 1 | Surgical Outcomes of Tubular Ureteroplasty. Representative data from Pigs 1-5.

\begin{tabular}{|c|c|c|c|c|c|c|c|}
\hline \multirow[t]{2}{*}{ Animal } & \multirow{2}{*}{$\begin{array}{l}\text { Ureteroplasty approach and } \\
\text { stent strategy }\end{array}$} & \multicolumn{4}{|c|}{ Ultrasonography (hydronephrosis grade) } & \multirow{2}{*}{$\begin{array}{l}\text { Complications and } \\
\text { management }\end{array}$} & \multirow{2}{*}{$\begin{array}{l}\text { Terminal } \\
\text { outcomes }\end{array}$} \\
\hline & & 2 weeks & 4 weeks & 8 weeks & 12 weeks & & \\
\hline Pig 1 & Transperitoneal + double pigtail stent for 8 weeks & No & 2 & 2 & 4 & $\begin{array}{l}\text { Stent migration into the } \\
\text { bladder at } 4 \text { weeks post-op. } \\
\text { New stent reinserted until } \\
8 \text { weeks timepoint. }\end{array}$ & $\begin{array}{l}\text { Stricture found in } \\
\text { central neotissue. } \\
\text { Bulk scaffold } \\
\text { extruded into distal } \\
\text { ureteral lumen }\end{array}$ \\
\hline Pig 2 & $\begin{array}{l}\text { Retroperitoneal flank + double pigtail stent for } \\
12 \text { weeks }\end{array}$ & 1 & 2 & 1 & 1 & $\begin{array}{l}\text { Subcutaneous fistula } \\
\text { formation at } 2 \text { weeks }\end{array}$ & $\begin{array}{l}\text { Fistula formation in } \\
\text { central neotissue }\end{array}$ \\
\hline Pig 3 & Retroperitoneal flank + double pigtail stent for 8 weeks & No & 2 & 2 & 4 & None & $\begin{array}{l}\text { Stricture found in } \\
\text { central neotissue. } \\
\text { Bulk scaffold } \\
\text { extruded into distal } \\
\text { ureteral lumen }\end{array}$ \\
\hline Pig 4 & Retroperitoneal flank + double pigtail stent for 8 weeks & No & 1 & 1 & 4 & None & $\begin{array}{l}\text { Stricture found in } \\
\text { central neotissue. }\end{array}$ \\
\hline Pig 5 & Retroperitoneal flank + double pigtail stent for 8 weeks & No & 1 & 1 & 4 & None & $\begin{array}{l}\text { Stricture found in } \\
\text { central neotissue. } \\
\text { Bulk scaffold } \\
\text { extruded into implant } \\
\text { site. }\end{array}$ \\
\hline
\end{tabular}

continuity, kidney morphology, and indwelling catheter position. These analyses included ultrasonography (USG), RUPG and video-endoscopy (cystoscopy, ureterorenoscopy) as described in the following sections.

All animals were maintained for a total of 12 weeks and then sacrificed with an intravenous $0.2 \mathrm{ml} / \mathrm{kg}$ pentobarbital sodium and phenytoin sodium euthanasia solution (Euthasol; Virbac AH, Westlake, TX, United States). Following necropsy, reconstructed ureteral segments were excised from host tissues and divided axially into four circumferential rings ( 4-5 $\mathrm{mm}$ in length) representing two peripheral (adjacent to anastomotic border) and two central zones of neotissues. Tubular specimens from both central and peripheral regions were evaluated by histological, immunohistochemical (IHC), and histomorphometric analyses as well as for ex vivo assessment of functional responses to contractile/relaxing agents as detailed below. Tubular ureteral segments excised either $5 \mathrm{~cm}$ above or below the scaffold implantation site were evaluated in parallel as internal proximal controls (IPC) or internal distal controls (IDC), respectively. In addition, native ureteral tissues that were either excised to create the initial defect for graft implantation or harvested from the unoperated ureters were assessed similarly as nonsurgical controls (NSC).

\section{Urinary Tract Ultrasonography}

USG imaging was performed under general anesthesia by using Preirus Ultrasound system (Hitachi Aloka, Mitaka-shi, Tokyo, Japan) outfitted with a standard $10 \mathrm{~Hz}$ transducer probe to examine the right kidney parenchyma, hydronephrosis, perirenal collection, catheter position, and left kidney for reference. The severity of hydronephrosis was evaluated using the Onen hydronephrosis grading system on a scale from 0 to 4 as previously described (Onen, 2007).

\section{Cystoscopy, Retrograde Ureteropyelogram, and Ureterorenoscopy}

A Cysto-Urethro-Fiberoscope (Model no: 11,272 C; Karl Storz, Tuttlingen, Germany) was used for cystoscopy, catheter manipulations, and contrast studies. Under general anesthesia, swine were placed in supine position, scrubbed, and sterilely draped. The cystoscope was gently introduced into the bladder with saline infusion provided through the working channel. For contrast imaging, the distal right ureter was catheterized with a hybrid working wire Sensor $^{\mathrm{TM}}$; Boston Scientific, MA, United States) introduced through the working channel of the scope. A retrograde uretero-pyelogram [1:1 diluted iohexol contrast agent (Omniopaque 300; GE Healthcare, Milwaukee, WI, United States)] was performed with a five French open-ended catheter (Cook Urological Inc.; Bloomington, IN, United States) to demonstrate the renal collecting system with anterior/posterior and lateral images acquired with C-arm fluoroscopy (BV Pulsera; Philips, Eindhoven, Netherlands). Neotissues were identified with radiopaque markers positioned at the time of grafting. URS was performed at 12 weeks post-op prior to sacrifice to examine the extent of mucosal healing by using a flexible ureterorenoscope (Flex-X2S; Karl Storz, Tuttlingen, Germany). Video and still images were captured by a video processor system (Image 1 HUB; Karl Storz, Tuttlingen, Germany).

\section{Ex vivo Tissue Contractility and Relaxation Responses}

Ureteral tissue specimens ( $N=4$ per group) were excised from the peripheral and central regions of BLSF graft sites and IPC segments at 12 weeks post-op and ex vivo contractility/ relaxation responses were evaluated and compared to NSC as 


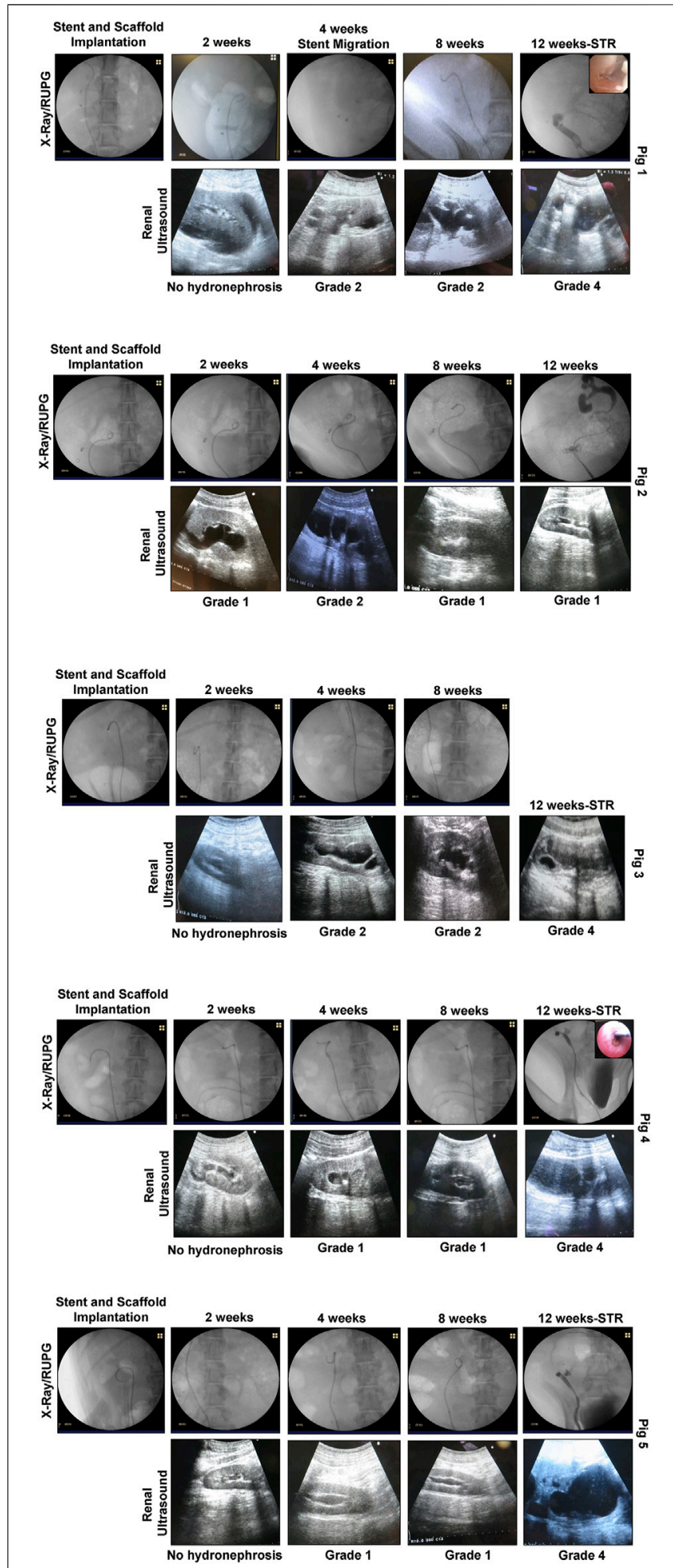

FIGURE 2 | In situ imaging evaluations of reconstructed ureteral conduits. Representative data for Pigs 1-5. Ureters were implanted with stented, tubular BLSF matrices. Stents were removed at 8 weeks post-op and swine were harvested at 3 months. In the majority of swine, severe Grade 4 hydronephrosis and neotissue stricture formation were detected in operated ureters at sacrifice. X-Ray (2, 4, 8 weeks) and contrast RUPG

(Continued) follows. After tissues were dissected into strips (6-8 $\mathrm{mm}$ in length and $2-3 \mathrm{~mm}$ in width) and denuded of mucosa, specimens were transferred to $5 \mathrm{ml}$ organ chambers containing Kreb's solution maintained at $37^{\circ} \mathrm{C}$ by a thermoregulated water circuit and continuously bubbled with a mixture of $95 \% \mathrm{O}_{2}$ and $5 \% \mathrm{CO}_{2}$ ( $\mathrm{pH}$ at 7.4). Each strip was tied at both ends by silk threads and connected to a force-displacement transducer to measure isometric tension from the circular muscle layer. Tissue samples were stretched to a force of $2 \mathrm{~g}$ and equilibrated for at least $120 \mathrm{~min}$. After the equilibration period, amplitude and frequency of spontaneous rhythmic contractions were recorded for a period of $30 \mathrm{~min}$ (baseline conditions). Increasing doses of phenylephrine (PE, 0.1-300 $\mathrm{M})$ were administered to the chambers and the resulting phasic contractions (amplitude and frequency) were measured. Following the last concentration of $\mathrm{PE}$, a single dose of isoproterenol (ISO, $10 \mu \mathrm{M})$ was added to provoke relaxation. The amplitude of contractile responses to the muscarinic receptor agonist, carbachol (CCh, $10 \mu \mathrm{M})$, was also evaluated. Receptor independent, smooth muscle contractions were elicited by exposure to a $120 \mathrm{mM} \mathrm{KCl}$ solution. Electrical field stimulation $(30 \mathrm{~V}, 1 \mathrm{~ms}$ duration, $1-20 \mathrm{~Hz})$, provided by parallel platinum electrodes embedded in the organ bath, was delivered at $2 \mathrm{~min}$ intervals to generate frequency-response curves. At the end of the experiment, the length, width and weight of each strip were recorded to normalize the force measurements by tissue cross-sectional area.

\section{Histological, Immunohistochemical, and Histomorphometric Analyses}

Tubular ureteral specimens from central and peripheral regions of neotissues $(N=5)$, IPC $(N=5)$, IDC $(N=5)$, and NSC were excised from swine following sacrifice, fixed in $10 \%$ neutralbuffered formalin, subjected to alcohol dehydration, and embedded in paraffin. Specimens were sectioned $(5 \mu \mathrm{m})$, stained with Masson's Trichrome (MTS), digitally imaged across the entire section, and collagen content was determined with a color segmentation program in Image J using previously described protocols to quantify blue-stained color elements indicative of collagen deposition (Affas et al., 2019). Collagen content was calculated as the percentage of the blue stained area (collagen) per total field area evaluated and normalized to NSC levels.

Immunohistochemical (IHC) assessments were performed on parallel tissue sections after antigen retrieval in $10 \mathrm{mM}$ sodium citrate buffer ( $\mathrm{pH}$ 6.0) and incubation in blocking buffer consisting of phosphate-buffered saline with $5 \%$ fetal bovine serum, $1 \%$ bovine serum albumin, and $0.3 \%$ Triton $\mathrm{X}-100$ for $1 \mathrm{~h}$ at room temperature. Specimens were probed with the following

FIGURE 2 | (12 weeks) (top rows); sonograms (bottom rows with hydronephrosis scores); and URS (insets showing regenerated mucosa) assessments were performed at various experimental timepoints. STR, stricture and RUPG, retrograde ureteropyelogram. 


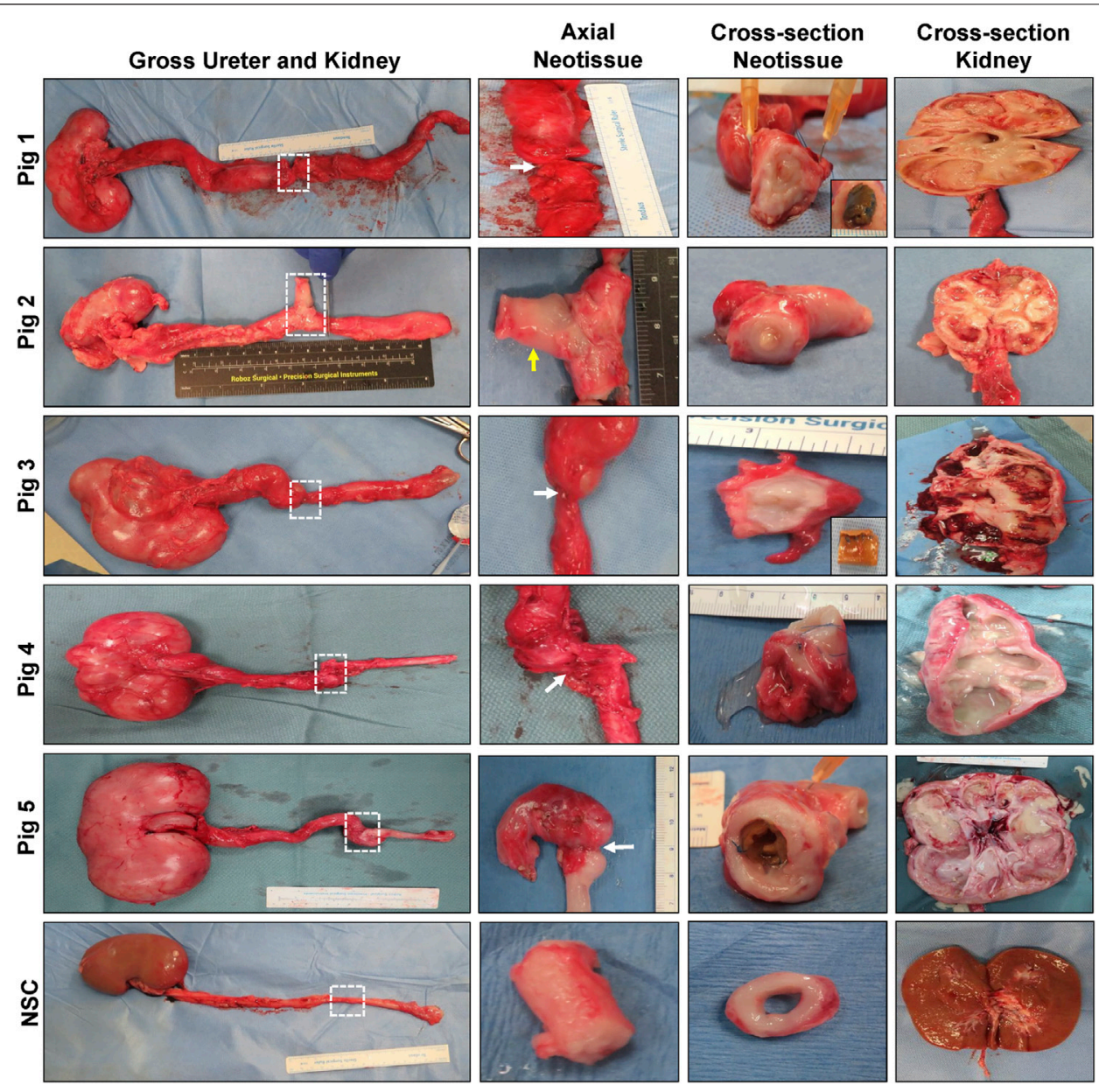

FIGURE 3 | Necropsy evaluations of ureteral neotissues and reconstructed collecting systems. First column: Photomicrographs of gross collecting systems and reconstructed areas (boxed) in Pigs 1-5 after 12 weeks of scaffold implantation as well as parallel nonsurgical controls (NSC). Second and third columns display axial and cross-sectional views of neoconduits from original graft sites, respectively. White arrows denote central stricture formation in neotissues and the yellow arrow marks fistula formation. Insets display bulk scaffold remnants isolated from the distal ureter. Cross-sectional view of kidneys from operated collecting systems or NSC are shown in the fourth column.

primary antibodies overnight at $4^{\circ} \mathrm{C}$ : anti-pan-cytokeratin $(\mathrm{CK})(1$ : 150 dilution; Dako, Carpinteria, CA), anti- $\alpha$-smooth muscle actin (SMA) (1:200 dilution; Sigma-Aldrich, St. Louis, MO), anti-SM22a (1:200 dilution, Abcam, Cambridge, MA), anti-S100 (1:200 dilution, Abcam), anti-uroplakin (UP) 3A (1:10 dilution, Fitzgerald, North Acton, MA), and anti-CD31 (1:100 dilution; Abcam). Specimens were then stained with species-matched Alexa Fluor 594-conjugated secondary antibodies (Thermo Fisher Scientific, Waltham, MA) and nuclei were counterstained with $4^{\prime}$, 6-diamidino-2-phenyllindole (DAPI). Specimen visualization was performed with a Zeiss Axio Imager M2 model (Carl Zeiss MicroImaging, Thornwood, NY) and representative fields were acquired with Zen software (version 3.1). Negative controls consisting of parallel specimens stained with secondary antibodies in the absence of primary antibodies were performed similarly and produced no detectable signal above background.

Histomorphometric evaluations ( $N=5$ swine per cohort) were carried out on four independent microscopic fields
(10X magnification) equally spaced along the circumference of each reconstructed and control tubular specimen using previously published protocols (Affas et al., 2019). Specifically, area measurements and image thresholding were performed on microscopic fields with ImageJ software (version 1.47) to determine the percentage of tissue area stained for $a$-SMA, SM22 $\alpha$ and pan-CK per total field area evaluated. The number of $\mathrm{CD}_{3} 1^{+}$vessels were calculated across four independent microscopic fields (10X) per sample using similar methods and normalized to total field area to calculate vascular density.

\section{Statistical Analysis}

Statistical evaluations of quantitative data were carried out using the Kruskal-Wallis test in combination with post hoc Dunn's test for pairwise comparisons, considering a value of $p<0.05$ as significant. Quantitative data were displayed as mean \pm standard deviation (SD) or \pm standard error (SE) when specified. 
A
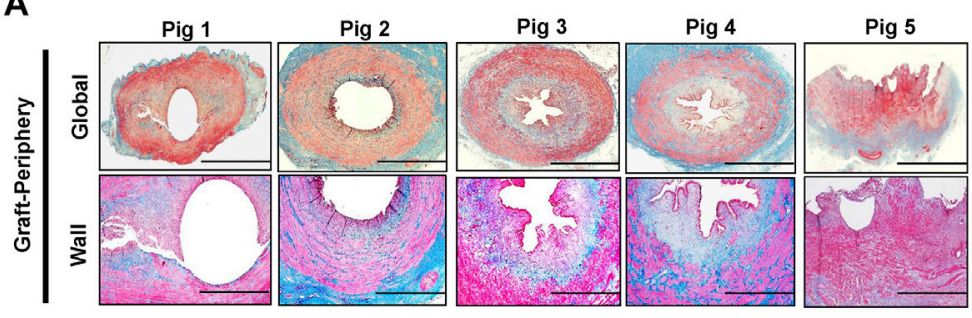

B
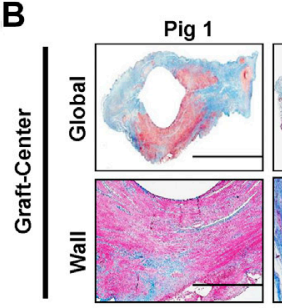

Pig 2

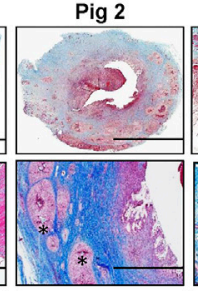

Pig 3

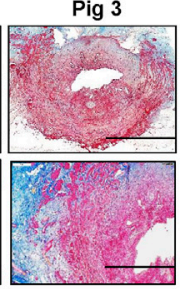

Pig 4

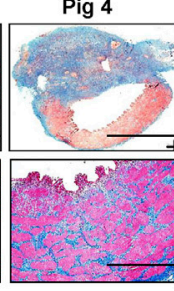

Pig 5

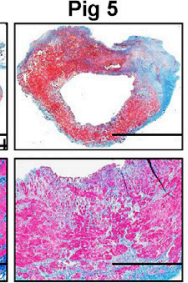

C
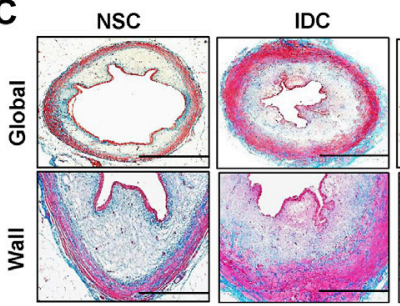

IPC

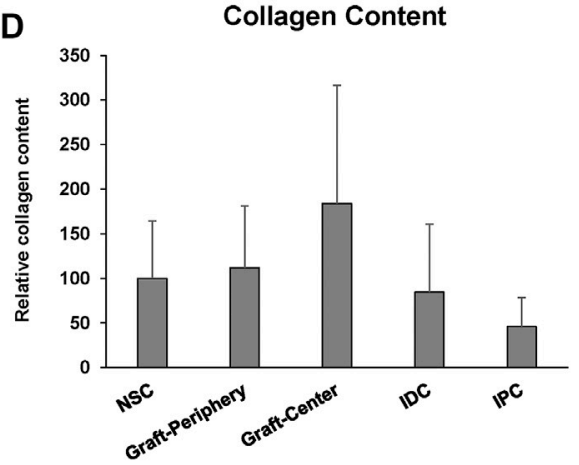

FIGURE 4 | Histological assessments of ureteral tissue architecture in controls and neotissues. Photomicrographs of peripheral (A) and central (B) areas of reconstructed tissues from all swine as well as control groups consisting of NSC, IPC, and IDC segments procured from Pig 5 (C) stained with Masson's trichrome. Gross cross-sections of ureteral tissues are presented in top rows with magnified views of the ureteral wall displayed in the second row of panels. Scale bars for $1^{\text {st }}$ and $2^{\text {nd }}$ rows in each panel are $1 \mathrm{~cm}$ and $400 \mu \mathrm{m}$, respectively. A semi-circumferential ureteral section is displayed for the graft-periphery of Pig 5. Asterisk denotes residual scaffold fragments in A-B. (D) Quantitation of collagen content in MTS-stained specimens displayed in (A-C). For all panels, $N=5$ animals per group were assessed per data point. For panel D, data were analyzed with Kruskal-Wallis test. $p=0.326$. Values are displayed as means \pm SD. NSC, nonsurgical controls; IPC, internal proximal controls; IDC, internal distal controls.

\section{RESULTS}

Tubular ureteroplasty with BLSF grafts was performed in five swine in combination with transient ureteral stenting of the implantation site to reinforce initial neoconduit integrity (Table 1). Anastomotic leak testing following ureteroplasty demonstrated a fluid-tight anastomosis during all five procedures. Fluoroscopic evaluations (Figure 2) confirmed the proximal coil of the double pigtail ureteral stent was properly positioned in the renal pelvis while the distal end was maintained in the bladder in each case. No signs of hydronephrosis were detected in any swine at time of ureteroplasty confirming normal anatomy of the collecting system. There were no intraoperative or immediate postoperative complications encountered in swine repaired with either the retroperitoneal flank or transperitoneal approach and all animals were successfully recovered from anesthesia.

At 1 week post-op, all swine had their Penrose drains and Foley catheters removed. There were no signs of surgical site infection or hydronephrosis at this timepoint and all animals were capable of normal micturition for the duration of the study following removal of the Foley catheter. Following 2 weeks of scaffold implantation, radiographic and sonographic assessments (Figure 2) on all five animals confirmed stent position in the urinary tract with no substantial evidence of hydronephrosis or renal abnormalities observed in any case. However, urine discharge was noted in Pig 2 from the subcutaneous closure area which was left to heal spontaneously. Imaging evaluations at 4 weeks post-ureteroplasty confirmed stent position in all swine except Pig 1 wherein the stent was dislodged into the urinary bladder. Cystoscopy was performed to remove the migrated stent from the bladder and a new stent was inserted as described above under radiographic guidance. Mild hydronephrosis (Grades 1-2) was observed in the reconstructed collecting systems of all animals at this timepoint with dilation of the renal pelvis, but no evidence of renal parenchymal damage. Urine drainage from the cutaneous wound closure area was still observed in Pig 2 in the absence of erythema, tenderness, or other signs of infection.

At 8 weeks post-op, ureteral stents were removed from the urinary tract in all swine except Pig 2 which displayed a persistent urine discharge from the primary surgical incision area. RUPG and fistulographic analyses confirmed the presence of an ureterocutaneous fistula in close proximity to the reconstructed site in Pig 2. Imaging evaluations (Figure 2) of all swine at this stage of repair revealed an unobstructed lumen at the graft site with mild hydronephrosis (Grades 1-2) of the reconstructed upper urinary tract observed in all cases similar to the findings at the 4 weeks timepoint. The ability of ureteral neotissues to support upper urinary tract function without stenting was assessed over the course of a 4 week period. At 12 weeks post-op, radiographic and sonographic assessments (Figure 2) demonstrated obstruction of the original graft area with severe hydronephrosis (Grade 4) in the reconstructed collecting systems of all unstented swine (Pigs 1, 3-5). In contrast, continuously stented ureteral tissue in Pig 2 


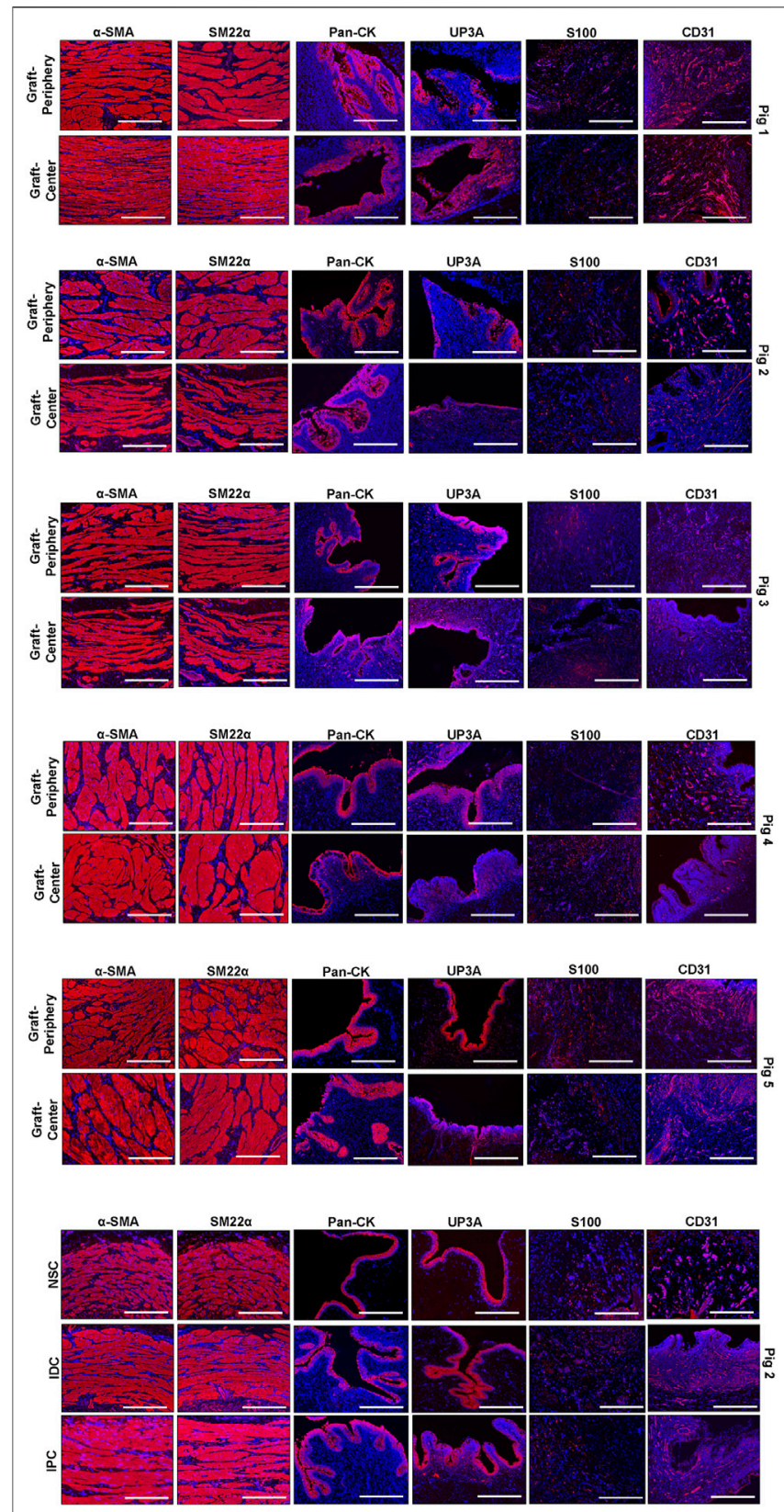

FIGURE 5 | Immunohistochemical evaluations of neconduit maturation and control tissues. Representative photomicrographs of selective protein expression in peripheral and central regions of neotissues at 12 weeks post-op as well as in control groups: NSC, IPC, and IDC. Markers of interest include smooth muscle contractile proteins ( $\alpha$-SMA, SM22 $\alpha$ ), epithelial proteins (pan-CK, UP3A), as well as vascular (CD31) and innervation (S100) proteins. For all panels, respective marker expression is labeled in red (Alexa Fluor 594 labeling) with DAPI nuclear counterstain displayed in blue. Scale bars $=400 \mu \mathrm{m}$ in each panel. Representative results for Pigs 1-5. Control specimens (NSC, IPC, and IDC) were procured from Pig 2. a-SMA, a-smooth muscle actin; CK, cytokeratin; DAPI, 4', 6-diamidino-2phenyllindole; UP3A, uroplakin 3A.

resulted in mild hydronephrosis (Grade 1) and free passage of contrast material through the neotissue. URS surveillance demonstrated mucosal regeneration at the distal graft region in the majority of animals, however stricture formation was apparent in the center of all neotissues except Pig 2.

Following scheduled euthanasia, necropsy assessments revealed host tissue ingrowth throughout the original implantation site in all swine with minimal abdominal adhesions observed on the exterior of neotissues (Figure 3). Regenerated ureteral tissues exhibited minimal axial contraction between the proximal/distal marking sutures, however the presence of strictures at the central graft site in Pigs 1, 3-5 confirmed URS findings. In addition, the formation of an uretero-cutaneous fistula was confirmed in the central neotissue region of Pig 2. Tubular BLSF grafts remained largely intact in the reconstructed collecting system and were either found extruded in the lumen of the graft site (Pig 5) or displaced into the unoperated segment of the distal ureter (Pigs 1, $3)$. Gross inspection of the reconstructed collecting systems confirmed imaging findings and demonstrated severe hydronephrosis in Pigs 1, 3-5 with substantial dilation of renal calyces and pelvis consistent with urine outflow obstruction. These results were in contrast with the unoperated collecting system which displayed normal anatomy.

Histological (MTS) evaluations (Figure 4) of study groups revealed that the cross-sectional organization of both peripheral and central neotissues demonstrated architecture similar to NSC with a multi-layer urothelium, a vascularized lamina propria, and an outer layer of smooth muscle bundles. However, in comparison to NSC, smooth muscle hypertrophy and hyperplasia were apparent in all reconstructed neotissues as well as in IPC and IDC segments consistent with compensatory mechanisms of ureteral obstruction. In addition, central neotissue regions displayed a discontinuous layer of smooth muscle bundles and substantially elevated levels of collagen content in respect to other experimental groups suggesting ongoing tissue remodeling. Moreover, scattered mononuclear inflammatory cells were also observed surrounding minute scaffold fragments in the center of neoconduits consistent with an active stage of wound healing.

Regenerated and control tissues were next evaluated by IHC (Figure 5) and histomorphometric (Figure 6) analyses to assess the degree of de novo tissue maturation achieved at implant sites. Pan-CK + epithelia were observed throughout the entire length of regenerated tissues with the level of immunoreactivity similar between all experimental cohorts. In addition, UP3A positivity was also evident in the apical layers of all regenerated epithelia providing evidence for the formation of mature urothelium in neoconduits. Smooth muscle bundles expressing contractile proteins, $a$-SMA and SM22a, were detected in both central and peripheral areas of the de novo ureteral wall in all specimens. However, the expression pattern of these markers was discontinuous along the circumference of the neotissue interior suggesting muscle formation was underdeveloped at this timepoint. Relative immunoreactivity of $a$-SMA and SM22a was found to be significantly elevated in the graftperiphery, IPC and IDC segments in respect to the NSC group consistent with histological findings of smooth muscle hypertrophy and hyperplasia secondary to ureteral obstruction. De novo innervation and vascularization processes were also 

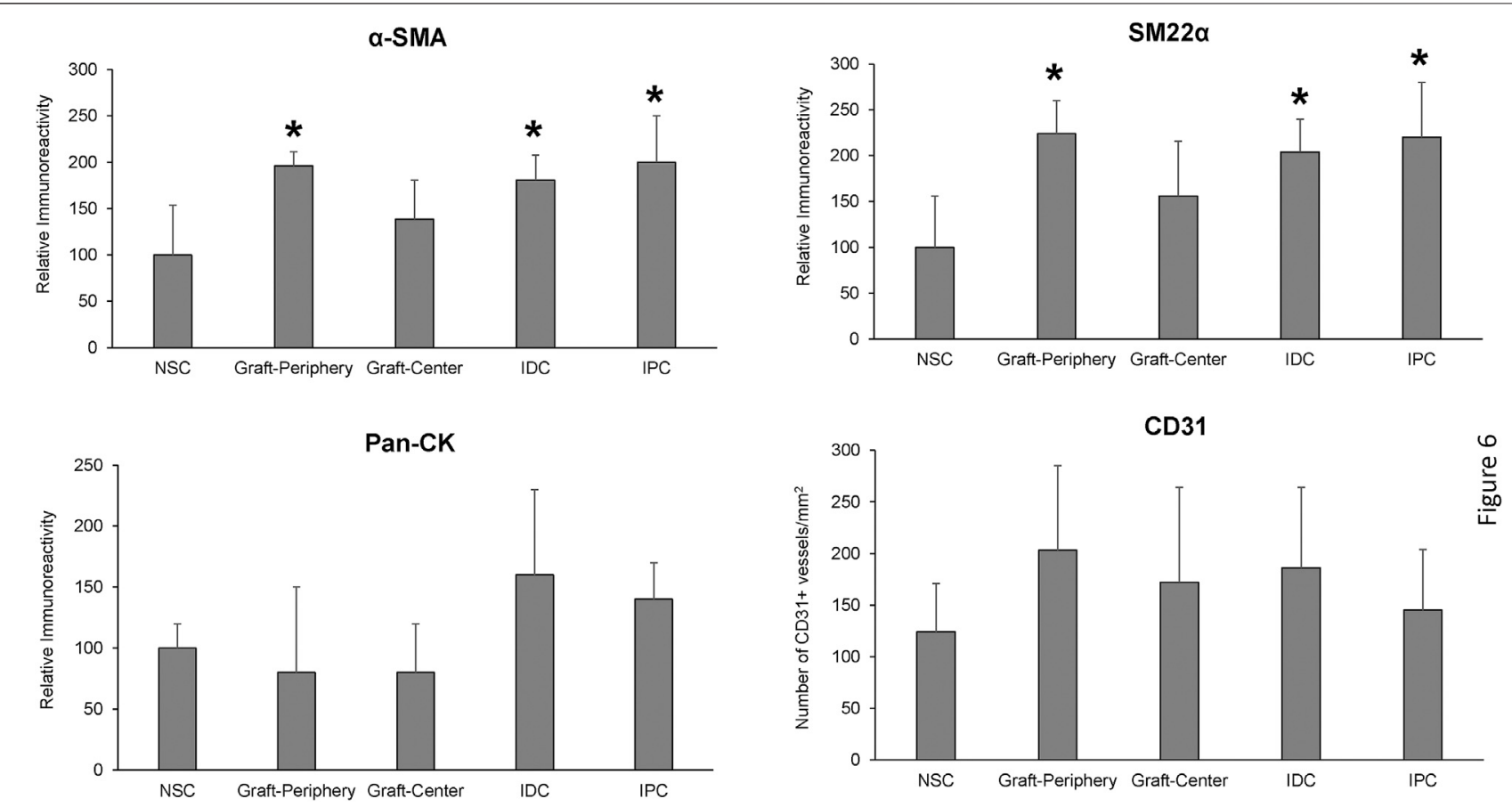

FIGURE 6 | Histomorphometric evaluations of tissue-specific proteins in neoconduits and control groups. Quantitative analyses of marker expression in neotissue regions at 12 weeks post-op and control cohorts following immunohistochemical staining. Values are presented as means \pm SD. NSC, nonsurgical controls; IPC, internal proximal controls; IDC, internal distal controls. Results from all groups were analyzed with Kruskal-Wallis and post hoc Dunn's tests. ${ }^{\star} p<0.05$ relative to respective NSC levels. $N=5$ samples per data point from all swine.

apparent in all neotissues as characterized by the presence of $\mathrm{S} 100+$ nerve fibers and blood vessels lined with $\mathrm{CD} 31^{+}$ endothelial cells, respectively. Taken together, these results demonstrate that BLSF grafts support the formation of innervated, vascularized ureteral tissues with urothelial and smooth muscle components.

Ex vivo tissue bath analyses were performed to quantify the contractile/relaxation responses of control and regenerated ureteral tissues (Figure 7). Following tissue equilibration, spontaneous rhythmic contractions were observed in all experimental segments except NSC. The frequency of spontaneous activity was significantly higher in IPC specimens compared to NSC under baseline conditions, although the amplitude of spontaneous activity was not different among segments (Figures 7A,B). Following administration of phenylephrine, rhythmic contractions became prominent in ureteral tissues from all segments, while the exposure to ISO reduced these contractions in each segment (Figures 7A,B). Ureteral tissue responded to $\mathrm{CCh}$ (Figure 7C) and $\mathrm{KCl}$ (Figure 7D) stimulation with a sustained contraction in all segments. Nerve-mediated contractions of variable amplitudes were recorded in response to EFS in both control and reconstructed neotissues. Although the graft-periphery region generated less force compared to the other segments in response to $\mathrm{CCh}, \mathrm{KCl}$ and EFS, there were no significant differences in contractile responses among segments (Figure 7E). These data provide evidence that ureteral reconstruction with tubular BLSF grafts leads to functional smooth muscle formation with contractile and relaxation properties.

\section{DISCUSSION}

Major ureteral reconstruction necessitates the use of tissue replacements to repair damaged ureteral segments and preserve upper urinary tract continuity. The goal of this study was to investigate the ability of BLSF grafts to promote functional repair of tubular ureteral defects in a porcine model. We chose to evaluate acellular scaffold configurations for ureteral tissue engineering since they offer particular advantages for clinical translation in comparison to cell-seeded counterparts including "off-the-shelf" utility and lack of secondary surgeries for procurement of patient-derived cells for construct seeding. Adult swine were used as a model species since the porcine ureter is of sufficient diameter to accommodate scaffold anastomosis, organ anatomy is similar to humans, and developmentally mature animals display minimal growthrelated changes in ureteral dimensions during the experimental period (Greca et al., 2004; de Jonge et al., 2015).

Overall, our data provide evidence that tubular BLSF conduits in combination with transient stenting support intrinsic regenerative processes sufficient to produce innervated, vascularized ureteral tissues with contractile/relaxation 
A Amplitude of Rhythmic Contractions

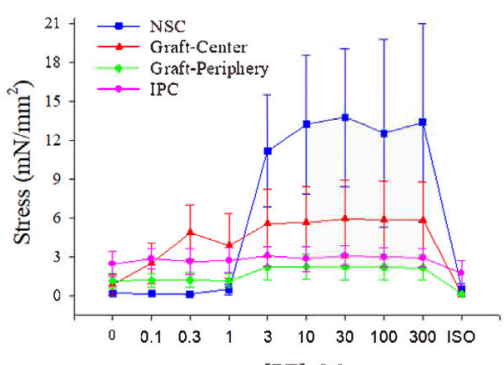

$[\mathrm{PE}] \mu \mathrm{M}$
B Frequency of Rhythmic Contractions

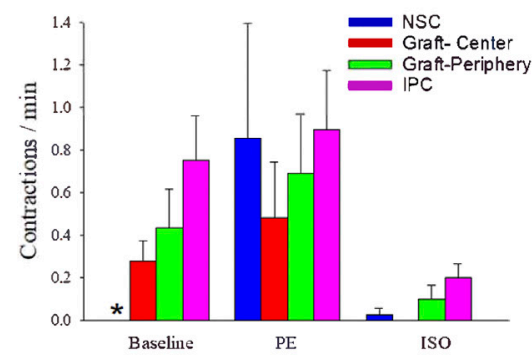

C Amplitude of CCh contractions

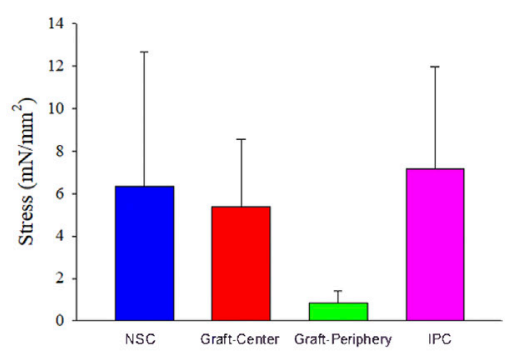

D

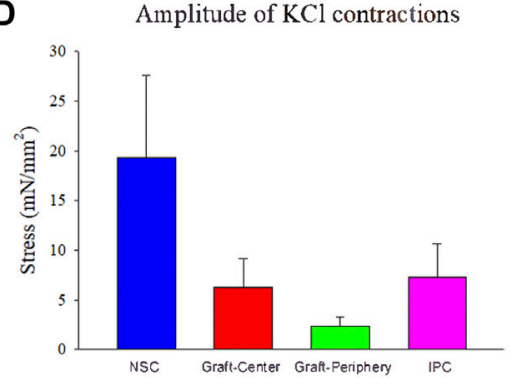

E

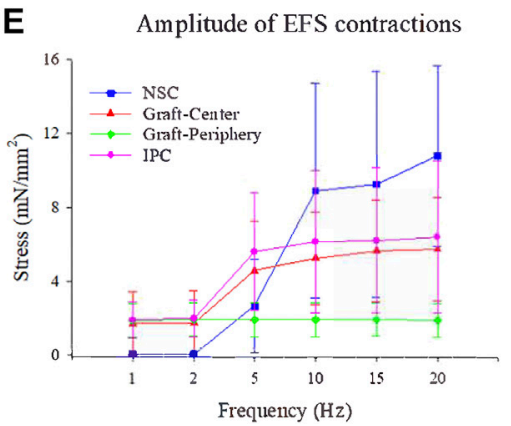

FIGURE 7 | Ex vivo contractile and relaxation responses in experimental groups. Quantitative assessments of contractile and relaxation responses in ureteral muscle tissue from NSC (blue bars and plots), graft-center (red bars and plots), graft-periphery (green bars and plots), and IPC segments at 12 weeks post-op. (A) The amplitude of rhythmic activity was measured before and after administration of increasing concentrations of phenylephrine (PE) followed by isoproterenol (ISO, $10 \mu \mathrm{M})$. (B) The frequency of spontaneous activity under baseline conditions, as well as the frequency of evoked activity after PE (300 $\mu \mathrm{M})$ and (ISO, $10 \mu \mathrm{M})$, were compared among each segment. (C-E) The amplitude of ureteral evoked responses induced by (C) carbachol (CCh, $10 \mu \mathrm{M})$, (D) $\mathrm{KCl}(120 \mathrm{mM})$, and (E) electrical field stimulation (EFS). For all panels, values are displayed as means \pm SE with $N=4$ per data point including specimens from Pigs $1-4$. Results from all segments were analyzed with Kruskal-Wallis and post hoc Dunn's tests. ${ }^{*} p<0.05$ relative to respective IPC levels in (B). NSC, nonsurgical controls; IPC, internal proximal controls.

functionality. However, a major limitation of the current scaffold design and surgical approach was the development of severe hydronephrosis, hydroureter and renal damage secondary to ureteral obstruction following stent removal. Ureteral occlusion was a consequence of stricture formation in central regions of neoconduits as well as extrusion and poor degradability of bulk scaffold remnants into the distal ureter. These results are supported by observations that ureteral dilation was present in both proximal and distal segments relative to the original implantation site in the majority of swine. Transient stenting of tubular tissue engineered constructs has been previously used to mitigate stenosis and hydronephrosis in reconstructed ureters presumably by reinforcing the mechanical integrity of the neotissue lumen during early stages of tissue remodeling (Zhang et al., 2012; Liao et al., 2013). However, histological and IHC evaluations confirmed that central neotissue areas supported by BLSF grafts were immature at sacrifice relative to peripheral neotissues and NSC suggesting longer periods of stenting may be necessary for complete regeneration and stricture prevention. Indeed, the duration of stent deployment has been shown to play a major role in determining the risk of stricture occurrence in reconstructed visceral hollow organs (Takimoto et al., 1994; Gundogdu et al., 2021). In addition, the in vivo degradation profile of BLSF matrices was also found to be insufficient for maintaining upper urinary tract function following stent removal.

Previous in vivo studies of porous silk foams have demonstrated that the initial silk fibroin content and pore size significantly influence the rate of scaffold degradation and degree of host tissue ingrowth (Wang et al., 2008). These data suggest that the degradation rate of the foam compartment of BLSF grafts may be increased by reducing the concentration of silk fibroin used during casting or by enhancing pore size via modulation of salt porogen diameter. In addition, in vivo degradation kinetics of silk fibroin films have been reported to be significantly dependent on the degree of $\beta$-sheet crystallinity which can be temperature controlled during water vapor annealing processes (Ghezzi et al., 2016). Future experiments will focus on optimization of BLSF scaffold fabrication parameters to enhance degradation kinetics by implementing selective changes in the casting process for both the film and foam layers. Specifically, we predict that reductions in the initial silk fibroin content of the foam compartment in combination with increases in pore size via modulation of porogen diameter will result in more rapid in vivo degradation of the BLSF graft. We also anticipate that bulk BLSF matrix degradation can be further improved by reducing the level of $\beta$-sheet crystallinity in the film layer by deploying water vapor 
annealing at $25^{\circ} \mathrm{C}$ for film construction as previously reported (Ghezzi et al., 2016).

Differential wound healing outcomes between peripheral and central neotissue areas suggest that de novo tissue formation occurs from ingrowth of host ureteral tissues adjacent to the anastomotic borders of the implantation site. Host tissues appear to primarily propagate along the exterior of the graft wall from both proximal and distal directions toward the interior resulting in luminal extrusion of the bulk matrix while promoting defect consolidation. By utilizing the film layer of BLSF grafts as a substrate, the invading neomucosa is potentially shielded from noxious urinary components in the foam compartment until epithelialization is complete. Indeed, BLSF grafts were capable of promoting multi-layered, urothelial formation in neotissues with prominent UP3A and pan-CK protein expression. This pattern of constructive remodeling is similar to our previous observations using BLSF scaffolds for bladder and esophageal tissue repair (Affas et al., 2019; Gundogdu et al., 2021).

Ureteral peristalsis plays a crucial role in transporting urine from the renal pelvis toward the bladder for storage and expulsion. Ureteral neoconduits supported by BLSF grafts contained circular smooth muscle layers with the ability to produce spontaneous and phenylephrine-induced, rhythmic contractions that could be attenuated by isoproterenol. These findings demonstrate myogenic activity in the neotissues that may underlie peristalsis in situ, and functional $a$ - and $\beta$ adrenoceptors in regenerated smooth muscle that can modulate urine propulsion. Regenerated tissues also showed a propensity to contract in response to $\mathrm{KCl}$ and $\mathrm{CCh}$ stimulation, indicating a fully intact contractile machinery capable of activation by membrane depolarization and muscarinic receptor signaling, respectively. Our findings also revealed that neoconduits were capable of contracting in response to EFS, thus indicating that excitatory neurotransmitters are released from functionally intact intrinsic nerves in implant regions.

\section{CONCLUSION}

The findings from our current report reveal that acellular, tubular BLSF scaffolds are permissive for ureteral tissue formation when deployed in tandem with transient stenting. All neotissues were composed of vascularized, innervated epithelial and muscular components capable of contractile and relaxation responses with minimal inflammatory reactions. Stented neoconduits were able to maintain upper urinary tract function during initial stages of implantation. However, future improvements in our graft technology are necessary to mitigate stricture formation and enhance in vivo scaffold degradation following stent removal to prevent longterm ureteral obstruction. In summary, BLSF biomaterials represent emerging platforms for tubular ureteroplasty and may offer a functional replacement for conventional autologous grafts following further optimization.

\section{DATA AVAILABILITY STATEMENT}

The raw data supporting the conclusions of this article will be made available by the authors, without undue reservation.

\section{ETHICS STATEMENT}

The animal study was reviewed and approved by All animal husbandry, surgical manipulations, and imaging evaluations followed guidelines prescribed by the National Institutes of Health's Guidelines for the Care and Use of Laboratory Animals and were reviewed and approved by the University of California, Irvine Animal Care and Use Committee and performed under protocol AUP-19-166. This study was also conducted in compliance with ARRIVE guidelines (https:// arriveguidelines.org).

\section{AUTHOR CONTRIBUTIONS}

JM and GG conceived the study. JM, GG, HO, and SS developed the study design as well as wrote and edited the animal protocol. GG, ZO, SS, PJ, and HO performed surgical manipulations, acquired and evaluated radiographic and sonographic data, and provided animal husbandry. JM fabricated the BLSF grafts. GG, JM, FV, and SS acquired and analyzed histological, immunohistochemical, and histomorphometic data. VC and MS performed and analyzed ex vivo contractility/relaxation responses. $\mathrm{VC}$ and JM carried out statistical analyses. JM, GG, ZO, and VC wrote the article. All authors reviewed and edited the article for conceptual and technical content. JM coordinated and supervised all aspects of the study.

\section{FUNDING}

This research was supported by National Institutes of Health Grant, R01DK119240-02 as well as generous gifts from the Randy Douthit and Jerry D. Choate Tissue Engineering Funds. The funding sources had no role in study design; in the collection, analysis and interpretation of data; in the writing of the report; or in the decision to submit the article for publication.

\section{ACKNOWLEDGMENTS}

The authors acknowledge Dr. David Kaplan at the Tufts University, Tissue Engineering Resource Center for procurement of silkworm cocoons as well as Mr. Kyle Costa at Boston Children's Hospital for silk fibroin solution preparation. The authors also acknowledge Mr. Travis Nguyen for histologic technical assistance. 


\section{REFERENCES}

Affas, S., Schäfer, F.-M., Algarrahi, K., Cristofaro, V., Sullivan, M. P., Yang, X., et al. (2019). Augmentation Cystoplasty of Diseased Porcine Bladders with Bi-layer Silk Fibroin Grafts. Tissue Eng. A 25 (11-12), 855-866. doi:10.1089/ ten.TEA.2018.0113

Algarrahi, K., Affas, S., Sack, B. S., Yang, X., Costa, K., Seager, C., et al. (2018a). Repair of Injured Urethras with Silk Fibroin Scaffolds in a Rabbit Model of Onlay Urethroplasty. J. Surg. Res. 229, 192-199. doi:10.1016/j.jss.2018.04.006

Algarrahi, K., Franck, D., Cristofaro, V., Yang, X., Savarino, A., Affas, S., et al. (2018b). Bi-layer Silk Fibroin Grafts Support Functional Tissue Regeneration in a Porcine Model of Onlay Esophagoplasty. J. Tissue Eng. Regen. Med. 12 (2), e894-e904. doi:10.1002/term.2402

Blackwell, R. H., Kirshenbaum, E. J., Shah, A. S., Kuo, P. C., Gupta, G. N., and Turk, T. M. T. (2018). Complications of Recognized and Unrecognized Iatrogenic Ureteral Injury at Time of Hysterectomy: A Population Based Analysis. J. Urol. 199 (6), 1540-1545. doi:10.1016/j.juro.2017.12.067

Burks, F. N., and Santucci, R. A. (2014). Management of Iatrogenic Ureteral Injury. Ther. Adv. Urol. 6 (3), 115-124. doi:10.1177/1756287214526767

Chung, Y. G., Algarrahi, K., Franck, D., Tu, D. D., Adam, R. M., Kaplan, D. L., et al. (2014a). The Use of Bi-layer Silk Fibroin Scaffolds and Small Intestinal Submucosa Matrices to Support Bladder Tissue Regeneration in a Rat Model of Spinal Cord Injury. Biomaterials 35 (26), 7452-7459. doi:10.1016/ j.biomaterials.2014.05.044

Chung, Y. G., Tu, D., Franck, D., Gil, E. S., Algarrahi, K., Adam, R. M., et al. (2014b). Acellular Bi-layer Silk Fibroin Scaffolds Support Tissue Regeneration in a Rabbit Model of Onlay Urethroplasty. PLoS One 9 (3), e91592. doi:10.1371/ journal.pone.0091592

de Jonge, P. K. J. D., Simaioforidis, V., Geutjes, P. J., Oosterwijk, E., and Feitz, W. F. J. (2015). Recent Advances in Ureteral Tissue Engineering. Curr. Urol. Rep. 16 (1), 465. doi:10.1007/s11934-014-0465-7

Duchene, D. A., Jacomides, L., Ogan, K., Lindberg, G., Johnson, B. D., Pearle, M. S., et al. (2004). Ureteral Replacement Using Small-Intestinal Submucosa and a Collagen Inhibitor in a Porcine Model. J. Endourology 18 (5), 507-511. doi:10.1089/0892779041271472

El-Hakim, A., Marcovich, R., Chiu, K.-Y., Lee, B. R., and Smith, A. D. (2005). First Prize: Ureteral Segmental Replacement Revisited. J. Endourology 19 (9), 1069-1074. doi:10.1089/end.2005.19.1069

Engel, O., Rink, M., and Fisch, M. (2015). Management of Iatrogenic Ureteral Injury and Techniques for Ureteral Reconstruction. Curr. Op Urol. 25 (4), 331-335. doi:10.1097/MOU.0000000000000175

Ghezzi, C. E., Wang, L., Behlau, I., Rnjak-Kovacina, J., Wang, S., Goldstein, M. H., et al. (2016). Degradation of Silk Films in Multipocket Corneal Stromal Rabbit Models. J. Appl. Biomater. Funct. Mater. 14 (14), e266-e276. doi:10.5301/ jabfm. 5000274

Greca, F. H., Noronha, L., Bendhack, M., Feres, A., Soccol, A., and Duda, J. R. (2004). Use of Small Intestine Submucosa as Ureteral Allograft in Pigs. Int. Braz. J Urol. 30 (4), 327-335. doi:10.1590/\$1677-55382004000400013

Gundogdu, G., Morhardt, D., Cristofaro, V., Algarrahi, K., Yang, X., Costa, K., et al. (2021). Evaluation of Bilayer Silk Fibroin Grafts for Tubular Esophagoplasty in a Porcine Defect Model. Tissue Eng. Part A 27 (1-2), 103-116. doi:10.1089/ ten.TEA.2020.0061

Heijkoop, B., and Kahokehr, A. A. (2021). Buccal Mucosal Ureteroplasty for the Management of Ureteric Strictures: A Systematic Review of the Literature. Int. J. Urol. 28 (2), 189-195. doi:10.1111/iju.14426

Janke, H. P., de Jonge, P. K. J. D., Feitz, W. F. J., and Oosterwijk, E. (2019). Reconstruction Strategies of the Ureter and Urinary Diversion Using Tissue Engineering Approaches. Tissue Eng. B: Rev. 25 (3), 237-248. doi:10.1089/ ten.TEB.2018.0345

Kloskowski, T., Kowalczyk, T., Nowacki, M., and Drewa, T. (2013). Tissue Engineering and Ureter Regeneration: Is it Possible? Int. J. Artif. Organs 36 (6), 392-405. doi:10.5301/ijao.5000130

Kocot, A., Kalogirou, C., Vergho, D., and Riedmiller, H. (2017). Long-Term Results of Ileal Ureteric Replacement: a 25-Year Single-Centre Experience. BJU Int. 120 (2), 273-279. doi:10.1111/bju.13825

Liao, W., Yang, S., Song, C., Li, X., Li, Y., and Xiong, Y. (2013). Construction of Ureteral Grafts by Seeding Bone Marrow Mesenchymal Stem Cells and Smooth
Muscle Cells into Bladder Acellular Matrix. Transplant. Proc. 45 (2), 730-734. doi:10.1016/j.transproceed.2012.08.023

Liatsikos, E. N., Dinlenc, C. Z., Kapoor, R., Bernardo, N. O., Pikhasov, D., Anderson, A. E., et al. (2001). Ureteral Reconstruction: Small Intestine Submucosa for the Management of Strictures and Defects of the Upper Third of the Ureter. J. Urol. 165 (5), 1719-1723. doi:10.1016/S00225347(05)66401-410.1097/00005392-200105000-00088

Mauck, R. J., Hudak, S. J., Terlecki, R. P., and Morey, A. F. (2011). Central Role of Boari Bladder Flap and Downward Nephropexy in Upper Ureteral Reconstruction. J. Urol. 186 (4), 1345-1349. doi:10.1016/j.juro.2011.05.086

Meng, L.-C., Liao, W.-B., Yang, S.-X., Xiong, Y.-H., Song, C., and Liu, L.-Q. (2015). Seeding Homologous Adipose-Derived Stem Cells and Bladder Smooth Muscle Cells into Bladder Submucosa Matrix for Reconstructing the Ureter in a Rabbit Model. Transplant. Proc. 47 (10), 3002-3011. doi:10.1016/ j.transproceed.2015.10.035

Onal, B., Gultekin, M. H., and Simsekoglu, M. F. (2018). Preputial Graft Ureteroplasty for the Treatment of Complex Ureteral Stricture: A New Surgical Technique and Review of Literature. J. Endourology Case Rep. 4 (1), 136-139. doi:10.1089/cren.2018.0055

Onen, A. (2007). An Alternative Grading System to Refine the Criteria for Severity of Hydronephrosis and Optimal Treatment Guidelines in Neonates with Primary UPJ-type Hydronephrosis. J. Pediatr. Urol. 3 (3), 200-205. doi:10.1016/j.jpurol.2006.08.002

Packiam, V. T., Cohen, A. J., Pariser, J. J., Nottingham, C. U., Faris, S. F., and Bales, G. T. (2016). The Impact of Minimally Invasive Surgery on Major Iatrogenic Ureteral Injury and Subsequent Ureteral Repair during Hysterectomy: A National Analysis of Risk Factors and Outcomes. Urology 98, 183-188. doi:10.1016/j.urology.2016.06.041

Sack, B. S., Mauney, J. R., and Estrada, C. R., Jr. (2016). Silk Fibroin Scaffolds for Urologic Tissue Engineering. Curr. Urol. Rep. 17 (2), 16. doi:10.1007/s11934015-0567-x

Seth, A., Chung, Y. G., Gil, E. S., Tu, D., Franck, D., Di Vizio, D., et al. (2013). The Performance of Silk Scaffolds in a Rat Model of Augmentation Cystoplasty. Biomaterials 34 (20), 4758-4765. doi:10.1016/ j.biomaterials.2013.03.038

Simaioforidis, V., de Jonge, P., Sloff, M., Oosterwijk, E., Geutjes, P., and Feitz, W. F. J. (2013). Ureteral Tissue Engineering: Where Are We and How to Proceed? Tissue Eng. Part B: Rev. 19 (5), 413-419. doi:10.1089/ten.teb.2012.0737

Siram, S. M., Gerald, S. Z., Greene, W. R., Hughes, K., Oyetunji, T. A., Chrouser, K., et al. (2010). Ureteral Trauma: Patterns and Mechanisms of Injury of an Uncommon Condition. Am. J. Surg. 199 (4), 566-570. doi:10.1016/ j.amjsurg.2009.11.001

Smith, T. G., Gettman, M., Lindberg, G., Napper, C., Pearle, M. S., and Cadeddu, J. A. (2002). Ureteral Replacement Using Porcine Small Intestine Submucosa in a Porcine Model. Urology 60 (5), 931-934. doi:10.1016/s0090-4295(02) 01890-3

Takimoto, Y., Teramachi, M., Okumura, N., Nakamura, T., and Shimizu, Y. (1994). Relationship between Stenting Time and Regeneration of Neoesophageal Submucosal Tissue. ASAIO J. 40 (3), M793-M797. doi:10.1097/00002480199407000-00107

Tu, D. D., Chung, Y. G., Gil, E. S., Seth, A., Franck, D., Cristofaro, V., et al. (2013). Bladder Tissue Regeneration Using Acellular Bi-layer Silk Scaffolds in a Large Animal Model of Augmentation Cystoplasty. Biomaterials 34 (34), 8681-8689. doi:10.1016/j.biomaterials.2013.08.001

Vasudevan, V. P., Johnson, E. U., Wong, K., Iskander, M., Javed, S., Gupta, N., et al. (2019). Contemporary Management of Ureteral Strictures. J. Clin. Urol. 12 (1), 20-31. doi:10.1177/2051415818772218

Wang, Y., Rudym, D. D., Walsh, A., Abrahamsen, L., Kim, H.-J., Kim, H. S., et al. (2008). In Vivo Degradation of Three-Dimensional Silk Fibroin Scaffolds. Biomaterials 29 (24-25), 3415-3428. doi:10.1016/ j.biomaterials.2008.05.002

Xiong, S., Wang, J., Zhu, W., Yang, K., Ding, G., Li, X., et al. (2020). Onlay Repair Technique for the Management of Ureteral Strictures: A Comprehensive Review. Biomed. Res. Int. 2020, 1-11. doi:10.1155/2020/6178286

Xu, Q., Chen, C., Xu, Z., Chen, F., Yu, Y., Hong, X., et al. (2020). Ureteral Reconstruction with Decellularized Small Intestinal Submucosa Matrix for Ureteral Stricture: A Preliminary Report of Two Cases. Asian J. Urol. 7 (1), 51-55. doi:10.1016/j.ajur.2019.03.004 
Zhang, J., Gu, G.-L., Liu, G.-H., Jiang, J.-T., Xia, S.-J., Sun, J., et al. (2012). Ureteral Reconstruction Using Autologous Tubular Grafts for the Management of Ureteral Strictures and Defects: An Experimental Study. Urol. Int. 88 (1), 60-65. doi:10.1159/000335002

Conflict of Interest: JM is a co-inventor on the patented BLSF technology, paid consultant and co-founder of WeaveTech Corporation. All other authors report no proprietary or commercial interest in any product mentioned or concept discussed in this article.

The remaining authors declare that the research was conducted in the absence of any commercial or financial relationships that could be construed as a potential conflict of interest.
Publisher's Note: All claims expressed in this article are solely those of the authors and do not necessarily represent those of their affiliated organizations, or those of the publisher, the editors and the reviewers. Any product that may be evaluated in this article, or claim that may be made by its manufacturer, is not guaranteed or endorsed by the publisher.

Copyright () 2021 Gundogdu, Okhunov, Cristofaro, Starek, Veneri, Orabi, Jiang, Sullivan and Mauney. This is an open-access article distributed under the terms of the Creative Commons Attribution License (CC BY). The use, distribution or reproduction in other forums is permitted, provided the original author(s) and the copyright owner(s) are credited and that the original publication in this journal is cited, in accordance with accepted academic practice. No use, distribution or reproduction is permitted which does not comply with these terms. 